\title{
Combined molecular targeted drug therapy for EGFR and HER-2 in head and neck squamous cell carcinoma cell lines
}

\author{
NORIO KONDO $^{1}$, MAMORU TSUKUDA ${ }^{2}$, ATSUKO SAKAKIBARA ${ }^{2}$, \\ HIDEAKI TAKAHASHI ${ }^{2}$, HIROSHI HYAKUSOKU ${ }^{2}$, MASANORI KOMATSU ${ }^{2}$, \\ TATSUO NIHO $^{2}$, KOUICHI NAKAZAKI ${ }^{2}$ and GABOR TOTH ${ }^{2}$ \\ ${ }^{1}$ Department of Otorhinolaryngology, Tama-Hokubu Medical Center, Tokyo; ${ }^{2}$ Department of Biology and \\ Function in Head and Neck, Yokohama City University Graduate School of Medicine, Yokohama, Japan
}

Received November 16, 2011; Accepted January 9, 2012

DOI: $10.3892 /$ ijo.2012.1376

\begin{abstract}
The epidermal growth factor receptor (EGFR) and related family member, HER-2, are often overexpressed simultaneously in patients with a variety of malignant tumors, and the combination may cooperatively promote cancer cell growth and survival. The purpose of this study was to examine antitumor effects of the combination treatment of cetuximab and trastuzumab on head and neck squamous cell carcinoma (HNSCC) using 16 HNSCC cell lines in terms of antiproliferative effect and antibody-dependent cell-mediated-cytotoxicity (ADCC). Previously we have reported the expression levels of EGFR mRNA on 16 HNSCC cell lines. All cell lines expressed mRNA for EGFR, HER-2 and HER-3; 12 cell lines expressed mRNA for HER-4; and 4 cell lines did not express mRNA for HER-4. In in vitro proliferation assay, the combination treatment of cetuximab and trastuzumab significantly lowered cell viability compared to either drug alone. The mRNA expression levels of EGFR and HER-2 were not correlated with the efficacy of the combination treatment of cetuximab and trastuzumab and the expression levels of HER-3 and HER-4 also showed no correlation with the efficacy of the combination treatment. We evaluated the gene status of HER-2 exons 23 and 24 in 16 HNSCC cell lines, but there was no mutation of HER-2 in any of the cell lines. Either drug showed ADCC in the 3 cell lines using peripheral blood mononuclear cells (PBMCs), however, a significant combination effect was not observed. Combined molecular targeted antibody drug therapy for EGFR and HER-2 may be useful in the treatment of HNSCC.
\end{abstract}

\section{Introduction}

Head and neck squamous cell carcinoma (HNSCC) is the fifth most common cancer and 500,000-900,000 HNSCC cases are

Correspondence to: Dr Norio Kondo, Department of Otorhinolaryngology, Tama-Hokubu Medical Center, 7-1, Aoba-cho 1 chome, Higashimurayama-shi, Tokyo 189-8511, Japan

E-mail: norio_kondo@tahamoku-hp.jp

Key words: EGFR, HER-2, cetuximab, trastuzumab newly diagnosed annually worldwide (1). The standard treatments for advanced HNSCC include surgery, radiotherapy and chemotherapy. These treatment modalities prolong the survival of individuals with advanced stage HNSCC; however, the treatment benefit is typically temporary in advanced-stage disease (2). Thus, novel and effective antitumor therapies, e.g., targeted molecular therapy, immunotherapy and gene therapy, are necessary for treating advanced HNSCC. Several new molecular-targeted drugs have been studied recently for HNSCC treatment and promising results have been obtained (3-8).

The epidermal growth factor receptor (EGFR) belongs to the human epidermal growth factor receptor (HER) family of receptor tyrosine kinases; this family includes EGFR (ErbB1 or HER-1), HER-2 (ErbB2), HER-3 (ErbB3) and HER-4 (ErbB4). EGFR overexpression has been frequently observed in HNSCC and is thought to be associated with carcinogenesis, metastasis, clinical stage of cancer, and poor prognosis $(9,10)$. HER-2 is a unique receptor since none of the known HER family ligands activate HER-2 homodimers (11). HER-2 appears to function primarily as a heterodimerization partner for other HER family members (12). HER-2 overexpression has been observed in HNSCC (13) and heterodimerization of HER-2 and EGFR is thought to mediate disease progression (14). High expression of EGFR and/or HER-2 has been associated with tumor cell resistance to chemotherapy and radiotherapy $(14,15)$. Therefore, EGFR and HER-2 are attractive targets for anticancer treatment.

Cetuximab (Erbitux ${ }^{\circledR}$ ) is a human/murine chimeric IgG1 monoclonal antibody that targets EGFR. Cetuximab binds to the EGFR with a 10-fold higher affinity than the natural ligand of EGFR and prevents ligand-induced phosphorylation in signal cascade pathways. In addition to those mentioned above, other mechanisms of cetuximab-mediated antitumor effect have been proposed. Cetuximab, as an IgG1 monoclonal antibody, exhibits different immunological effect and has potential antitumor activity. Preclinical results have shown that cetuximab can induce either complement-mediated tumor cell killing (CDC) or antibody-dependent cell-mediatedcytotoxicity (ADCC) by interacting with natural killer (NK) cells, monocytes and granulocytes by means of their specific Fc receptors $(16,17)$. 
Trastuzumab (Herceptin ${ }^{\circledR}$ ) is a humanized IgG1 monoclonal antibody against the extracellular domain of HER-2. Clinical trials have clearly shown that trastuzumab exhibits significant activity against metastatic breast cancer (18). The mechanisms of trastuzumab antitumor activity induce inactivation of signal transduction (19), the ability to disrupt the heterodimerization of HER-2 with other HER family members (11) and induce immunological responses such as $\operatorname{ADCC}(19,20)$.

We previously reported the antitumor effect of combined gefitinib and trastuzumab treatment on HNSCC in vitro (4). In this study, we aimed to determine whether combined cetuximab and trastuzumab treatment exerted antitumor effects, e.g., antiproliferative effect and ADCC, against HNSCC cell lines.

\section{Materials and methods}

Drug. Cetuximab (Erbitux ${ }^{\circledR}$ ) was provided by Merk Serono (Darmstadt, Germany). Trastuzumab (Herceptin ${ }^{\circledR}$ ) was purchased from Chugai Pharmaceutical Co., Ltd. (Tokyo, Japan). For the antiproliferative assays, cetuximab and trastuzumab were diluted with saline and dosing preparations of both agents were prepared on the day of use. Gefitinib (Iressa ${ }^{\circledR}$ ) was provided by AstraZeneca (Macclesfield, UK). Dosing preparations of all agents were prepared on the day of use.

Cell lines and culture conditions. Sixteen human HNSCC cell lines were examined in this study. The origins of these cell lines were the oral floor (YCU-OR891), hypopharynx (YCU-H891), mesopharynx (YCU-M862, KCC-M871, and YCU-M911), larynx (KCC-L871, YCU-L891), tongue (KCC-T871, KCC-T873, YCU-T891 and YCU-T892), and maxillary sinus (KCC-MS871, YCU-MS861), and metastatic tumors from different tongue carcinomas (KCC-TCM901, KCC-TCM902 and KCC-TCM903). These cell lines were established in the Department of Otolaryngology, Yokohama City University School of Medicine and Research Institute, Kanagawa Cancer Center. These cell lines were maintained in RPMI-1640 medium (Life technologies, Inc., Tokyo, Japan) supplemented with $10 \%$ fetal bovine serum (Gibco, Grand Island, NY), $2 \mathrm{mmol} / \mathrm{l} \mathrm{L-glutamine,} 100 \mathrm{U} / \mathrm{ml}$ penicillin and $100 \mu \mathrm{g} / \mathrm{ml}$ streptomycin. These cells were incubated at $37^{\circ} \mathrm{C}$ in a moist atmosphere containing $5 \% \mathrm{CO}_{2}$.

Detection of HER-2, HER-3 and HER-4 mRNAs by quantitative $R T-P C R$. Cells were grown until nearly confluent in RPMI-1640 supplemented with $10 \%$ FBS. Total RNA extracted from 17 human HNSCC cells with phenol solution (Isogen; Nippon Gene, Inc., Tokyo, Japan) according to the manufacturer's protocol. One microgram of total RNA was converted into cDNA by using Takara RNA PCR Kit (AMV) Ver.3.0 (Takara Bio, Inc., Tokyo, Japan). The quantification of relative expression levels of GAPDH and EGFR were carried out with TaqMan Gene Expression Master Mix (Applied Biosystems, Fostere City, CA) using an ABI Prism ${ }^{\circledR}$ 7500 Sequence Detection System (Applied Biosystems). PCR was conducted using the following cycle parameters: $50^{\circ} \mathrm{C}$ for $2 \mathrm{~min}, 95^{\circ} \mathrm{C}$ for $10 \mathrm{~min}$, followed by 60 cycles at $95^{\circ} \mathrm{C}$ for $15 \mathrm{sec}$ and $60^{\circ} \mathrm{C}$ for $1 \mathrm{~min}$. The primers used to detect each factor were as follows: GAPDH, Hs99999905 m1 (Applied Biosystems); HER-2, Hs01001580 m1 (Applied Biosystems);
HER-3, Hs00951455 m1 (Applied Biosystems); HER-4, Hs00171783 m1 (Applied Biosystems). The results were normalized by the corresponding GAPDH levels. Each PCR amplification was performed at least 3 times and the mean was calculated.

In vitro proliferation assay. Cell proliferation assay was performed to assess the effect of treatment on growth of the 16 human HNSCC cell lines. These cells were plated in 96-well flat plates (Sumilon; Sumitomo Bakekite Co., Ltd., Tokyo, Japan) at a concentration of $1 \times 10^{3}$ cells/well. In case of trastuzumab alone, after 72-h exposure to respective doses $(0.01,0.05,0.1$, 0.5 and $0.1 \mu \mathrm{M}$ ) of tastuzumab (5 wells of the 96 -well plate per experimental condition), the cell proliferation was assayed by incubating with Tetra Color One (Seikagaku Co., Ltd., Tokyo, Japan). Relative growth inhibition was calculated by dividing the number of recovered drug-treated cells by the number of vehicle-treated control cells. In case of the combination treatment of cetuximab and trastuzumab, after 72-h exposure to $10 \mathrm{nM}$ cetuximab and/or $0.1 \mu \mathrm{M}$ trastuzumab (5 wells of the 96-well plate per experimental condition), the cell proliferation was assayed and the relative growth inhibition was calculated by the same method of trastuzumab alone.

Detection of HER-2 mutations. Genomic DNA was extracted using SepaGene kit (Sanko Junyaku Co., Ltd., Tokyo, Japan). Exons 23 and 24 of HER-2 gene were amplified by polymerase chain reaction (PCR) using the following primer pairs: HER-2 exon 23, 5'-GCCCACGCTCTTCTCACTCA-3' and 5'-ATGGGGTCCTTCCTGTCCTC-3'; HER-2 exon 24, 5'-GTGATGGTTGGGAGGCTGTG-3' and 5'-GCTGCACC GTGGATGTCAG-3' (21-23). The PCR products were further analyzed by direct nucleotide sequencing using ABI PRISM 3100 DNA Analyzer (Applied Biosystems) in order to detect mutations.

Cytotoxic assay. Peripheral blood mononuclear cells (PBMC) were separated from peripheral blood obtained from healthy two donors (donor A and B) by centrifugation with BD Vacutainer ${ }^{\circledR}$ (Becton-Dickinson Co., Franklin Lakes, NJ). Cytotoxicity of PBMC cells were evaluated with $\mathrm{LDH}$ release experiments using CytoTox $96^{\circledR}$ Non-Radioactive Cytotoxicity Assays (Promega, Madison, WI) according to the procedure furnished by producers. Target cells were seeded at a concentration of $1 \times 10^{4}$ cells/well in 96 -well plates. Cetuximab at a concentration of $100 \mathrm{nM}$ and/or trastuzumab at a concentration of $1 \mu \mathrm{M}$ and effector cells of each effector/target cell ratio (E/T ratio) (40:1, 20:1 and 10:1) were added.

Cytotoxicity was evaluated with the following formula: $\%$ cytotoxicity $=($ experimental - effector spontaneous - target spontaneous)/(target maximum - target spontaneous) x100.

Statistical analysis. For statistical analyses, we used Student's paired t-test. Statistical significance was set at $\mathrm{p}<0.05$.

\section{Results}

Expression of HER-2, HER-3 and HER-4 mRNA in 16 HNSCC cell lines. Using real-time quantitative reverse transcriptasepolymerase chain reaction (RT-PCR), we analyzed the steady 


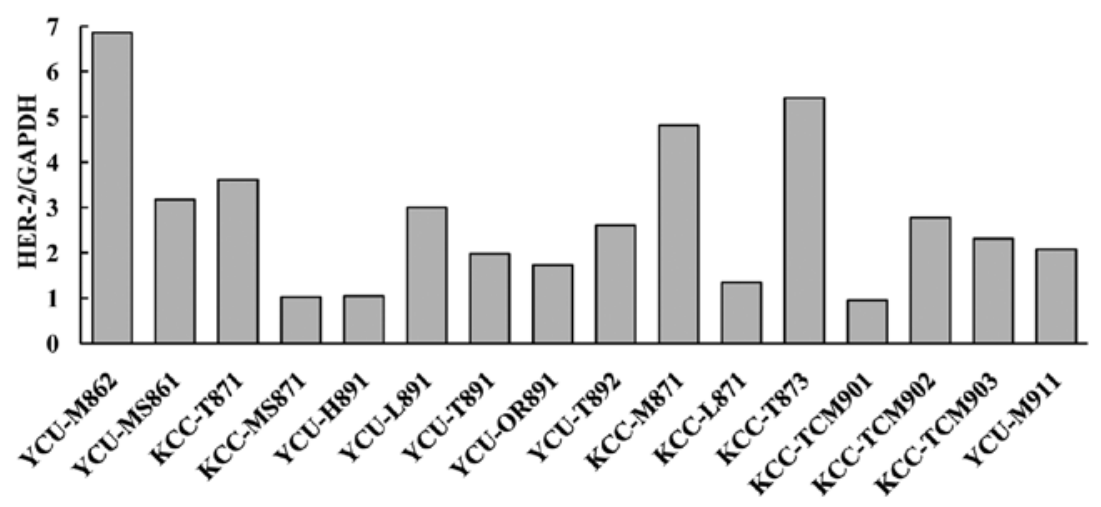

Figure 1. Expression of HER-2 mRNAs in HNSCC cell lines. Expression of mRNA was determined by real-time RT-PCR in 16 HNSCC cell lines. The level of each mRNA was normalized relative to GAPDH mRNA.

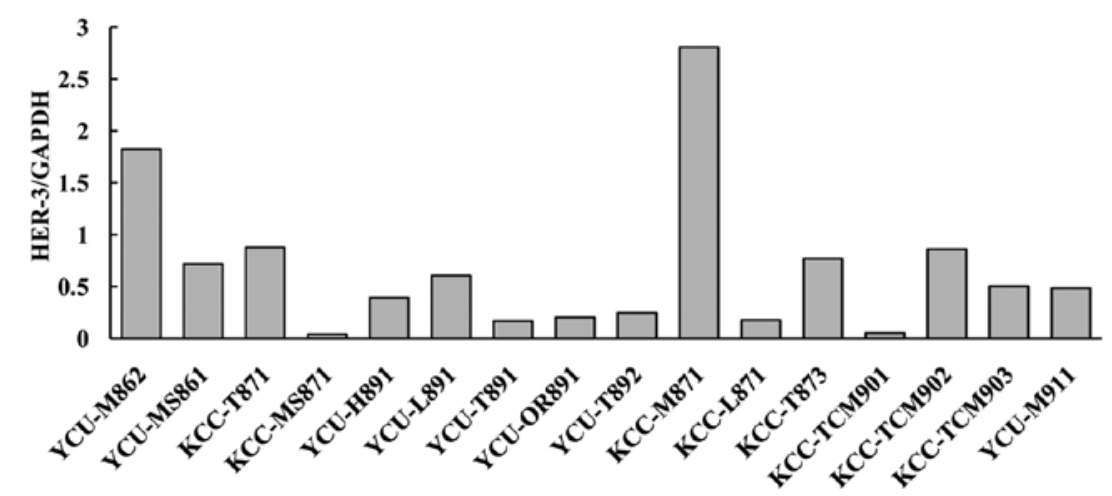

Figure 2. Expression of HER-3 mRNAs in HNSCC cell lines. Expression of mRNA was determined by real-time RT-PCR in 16 HNSCC cell lines. The level of each mRNA was normalized relative to GAPDH mRNA.

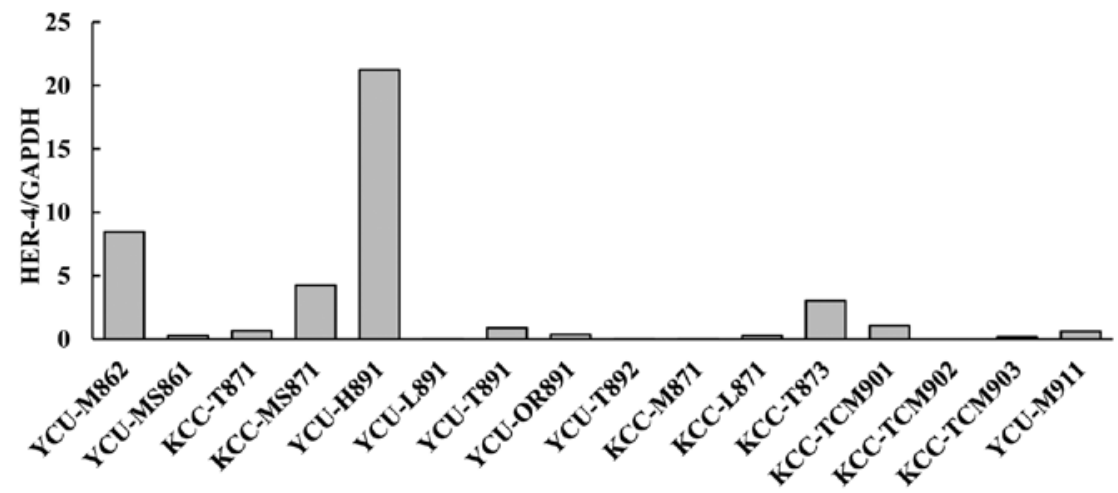

Figure 3. Expression of HER-4 mRNAs in HNSCC cell lines. Expression of mRNA was determined by real-time RT-PCR in 16 HNSCC cell lines. The level of each mRNA was normalized relative to GAPDH mRNA.

state levels of HER-2, HER-3 and HER-4 mRNA in 16 different HNSCC cell lines. The relative expression level of HER-2, HER-3 and HER-4 mRNA was calculated in comparison with the expression levels of glyceraldehyde-3-phosphate dehydrogenase (GAPDH). The expression of HER-2 and HER-3 mRNA varied widely, and all cell lines expressed mRNA encoding HER-2 and HER-3 (Figs. 1 and 2). On the other hand, only 12 cell lines expressed mRNA encoding HER- 4 and 4 cell lines did not express HER-4 mRNA (Fig. 3).
In vitro proliferation assay. We evaluated the antiproliferative effect of $0-1 \mu \mathrm{M}$ trastuzumab on HNSCC cell lines. As shown in Fig. 4, inhibition of trastuzumab-induced cell growth was $<25 \%$ in all cell lines and the cell viability of almost all cell lines reached a plateau at trastuzumab concentrations of $>0.1 \mu \mathrm{M}$.

Next, we examined the antiproliferative effect of combined cetuximab $(10 \mathrm{nM})$ and trastuzumab $(0.1 \mu \mathrm{M})$ treatment on 16 HNSCC cell lines. As shown in Table I, combined cetuximab 


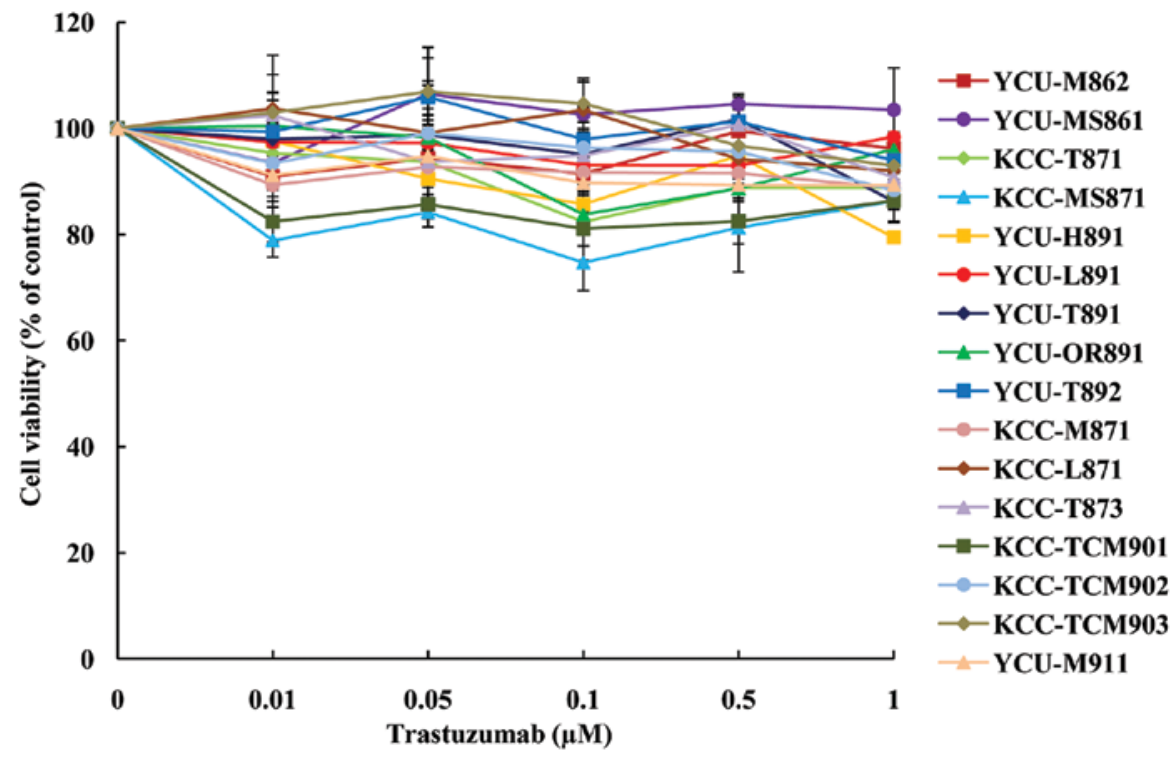

Figure 4. The antiproliferative activity of trastuzumab. In the proliferation assay described in Materials and methods, tumor cells were exposed to various concentrations of trastuzumab for $72 \mathrm{~h}$. Data are presented as mean values ( $\mathrm{n}=3$ per group), each was expressed as a percentage of cell growth relative to untreated controls. Error bars indicate SE.

Table I. Cell viability of the combination treatment of cetuximab and trastuzumab, and gene status of HER-2 in 16 HNSCC cell lines.

\begin{tabular}{|c|c|c|c|c|c|}
\hline \multirow[b]{2}{*}{ HNSCC cell lines } & \multirow[b]{2}{*}{ Primary site } & \multicolumn{3}{|c|}{ Cell viability ( $\%$ of control) } & \multirow[b]{2}{*}{ HER-2 exons 23,24} \\
\hline & & Cet & Tra & Cet+Tra & \\
\hline YCU-M862 & Mesopharynx & 92.7 & 101.4 & 83.8 & WT \\
\hline YCU-MS861 & Maxillary sinus & 89.7 & 102.1 & $75.9^{\mathrm{b}}$ & WT \\
\hline KCC-T871 & Tongue & 48.9 & 86.2 & $39.9^{\mathrm{b}}$ & WT \\
\hline KCC-MS871 & Maxillary sinus & 93.5 & 100.2 & 100.2 & WT \\
\hline YCU-H891 & Hypopharynx & 94.4 & 86.5 & $80.7^{\mathrm{b}}$ & WT \\
\hline YCU-L891 & Larynx & 86.9 & 105.7 & 80.4 & WT \\
\hline YCU-T891 & Tongue & 99.0 & 107.9 & $73.6^{\mathrm{b}}$ & WT \\
\hline YCU-OR891 & Oral floor & 83.9 & 106.4 & 81.4 & WT \\
\hline YCU-T892 & Tongue & 48.5 & 80.1 & 45.7 & WT \\
\hline КCC-M871 & Mesopharynx & 99.4 & 88.0 & 93.9 & WT \\
\hline KCC-L871 & Larynx & 102.8 & 94.6 & 94.8 & WT \\
\hline KCC-T873 & Tongue & 76.8 & 97.4 & $66.5^{\mathrm{b}}$ & WT \\
\hline КСC-ТCM901 & Lung $^{\mathrm{a}}$ & 89.8 & 95.3 & 86.5 & WT \\
\hline КCC-ТСМ902 & Lung $^{a}$ & 89.6 & 97.5 & $77.1^{\mathrm{b}}$ & WT \\
\hline КСС-ТCM903 & Lung $^{\mathrm{a}}$ & 95.2 & 104.7 & $70.9^{\mathrm{b}}$ & WT \\
\hline YCU-M911 & Mesopharynx & 76.6 & 91.8 & $70.8^{\mathrm{b}}$ & WT \\
\hline
\end{tabular}

${ }^{a}$ Metastatic tumors from different tongue carcinomas. Cet, cetuximab $10 \mathrm{nM}$; Tra, trastuzumab $0.1 \mu \mathrm{M}$; WT, wild-type. Cell viability of Cet+Tra is lower than cetuximab or trastuzumab alone. ${ }^{\mathrm{b}} \mathrm{p}<0.05$ as compared with cetuximab or trastuzumab alone.

and trastuzumab treatment significantly lowered cell viability (\% of control) as compared to treatment with cetuximab or trastuzumab alone in 8 HNSCC cell lines.

Mutation status of HER-2 in 16 HNSCC cell lines. We investigated the genomic DNA of 16 human HNSCC cell lines by direct sequencing of PCR products using primers designed to amplify the HER-2 exons 23 and 24. No mutation was observed in the HER-2 exons 23 and 24 (NM_001005862 in GenBank) (Table I).

Correlation between the antiproliferative effect of combined cetuximab and trastuzumab treatment and the mRNA expression of EGFR, HER-2, HER-3 and HER-4. We have 
A

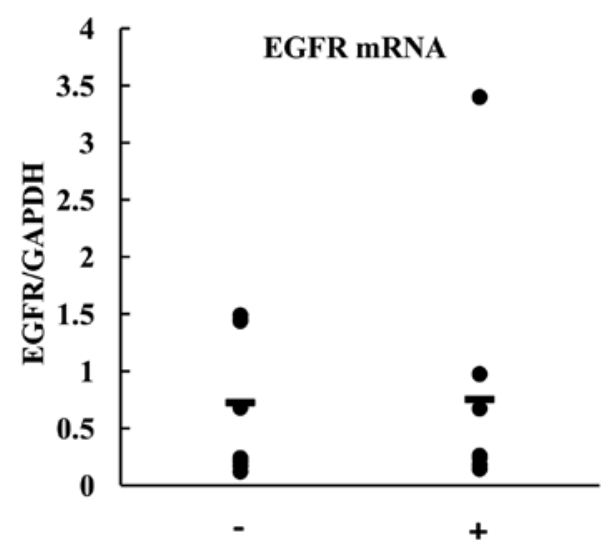

C

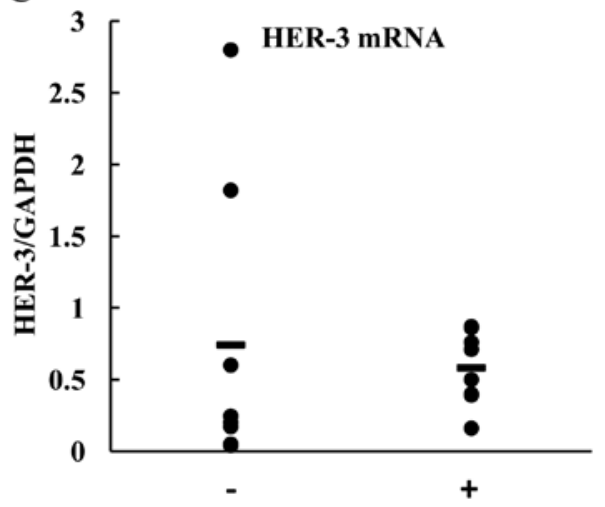

B

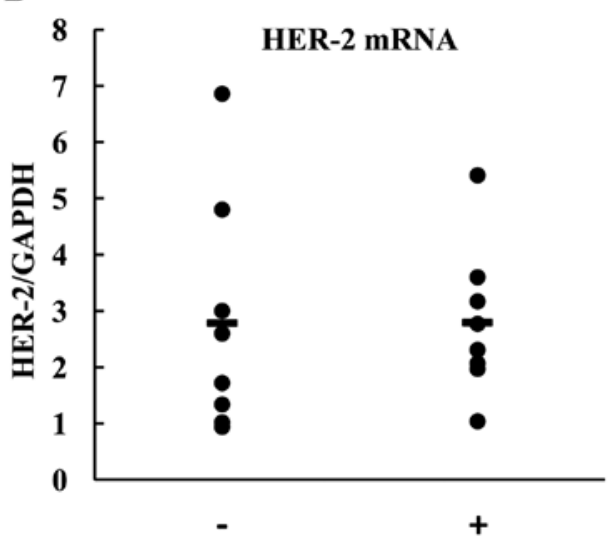

D

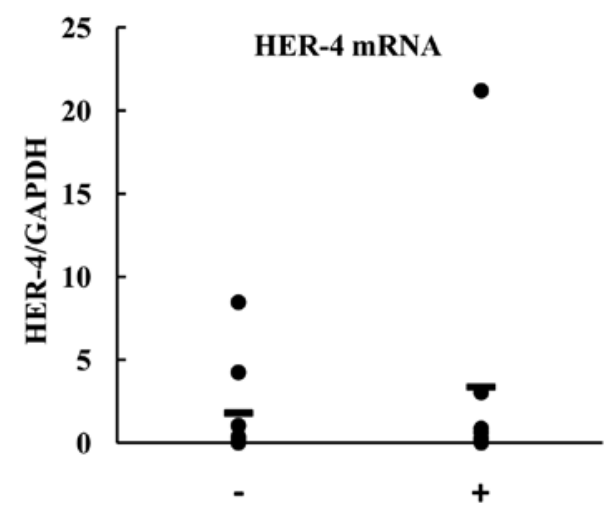

Figure 5. Correlation between the antiproliferative effect of the combination treatment of cetuximab and trastuzumab, and EGFR, HER-2, HER-3 and HER-4 mRNA expression. (A) Correlation with EGFR. EGFR mRNA levels were determined by quantitative RT-PCR as described (25). (B) Correlation with HER-2. (C) Correlation with HER-3. (D) Correlation with HER-4. Correlation was not observed between the antiproliferative effect of combination treatment of cetuximab and trastuzumab, and EGFR, HER-2, HER-3 and HER-4 mRNA expressions.

previously reported the expression levels of EGFR mRNA in the 16 HNSCC cell lines in a different study (24). To determine the correlation between the antiproliferative effect of combined cetuximab and trastuzumab treatment and the expression of EGFR, we analyzed the levels EGFR mRNA in 16 HNSCC cell lines (Figs. 1-3). On the basis of these results, we analyzed the correlation between HER-2, HER-3 and HER-4 expression and the antiproliferative effect of combined cetuximab and trastuzumab treatment. No correlation was observed (Fig. 5).

Cytotoxicity assay. We evaluated ADCC by peripheral blood mononuclear cells (PBMC) after treatment with cetuximab and trastuzumab alone and combined treatment with cetuximab and trastuzumab by performing lactate dehydrogenase (LDH) release experiments using EGFR high-, HER2 high-(KCCT873), EGFR low-, HER-2 moderate-(YCU-MS861), EGFR low- and HER-2 low-(YCU-T891)-expressing cells. PBMC used in the experiment were obtained from two healthy donors (donors A and B) (Figs. 6 and 7). Treatment with cetuximab and trastuzumab alone resulted in ADCC in all 3 cell lines in the case of both the donors. However, combined treatment with the drugs did not have a significant effect on all cell lines.

\section{Discussion}

Cetuximab has been approved by the food and drug administration (FDA) for clinical use in patients with colorectal cancer and HNSCC. Cetuximab has been established to be clinically effective in phase III trials of colorectal cancer and HNSCC patients; however only $10-20 \%$ of the treated patients responded to cetuximab. The primary mechanism of action of cetuximab may involve EGFR blockage. Cetuximab may block the binding of EGFR ligands, e.g., EGF and TGF- $\alpha$, and prevent ligand-dependent homodimerization, intracellular autophosphorylation and activation of intracellular cascades that control cellular proliferation, adhesion, angiogenesis and apoptosis $(25,26)$. In addition EGFR, HER-2 overexpression is also associated with poor prognosis of carcinoma patients. The humanized anti-HER-2 antibody trastuzumab is effective against metastatic breast cancer $(18,27)$. HER-2 appears to function primarily as a heterodimerization partner for other HER family members (12). EGFR heterodimers produce an intense and sustained proliferative signal that is greater than that of EGFR homodimers (28). Drug-targeting therapy against both EGFR and HER-2 is reported to be effective for breast cancer, non-small cell lung cancer, prostate cancer and vulvar squamous cell carcinoma (29-32). Further, we have 
A

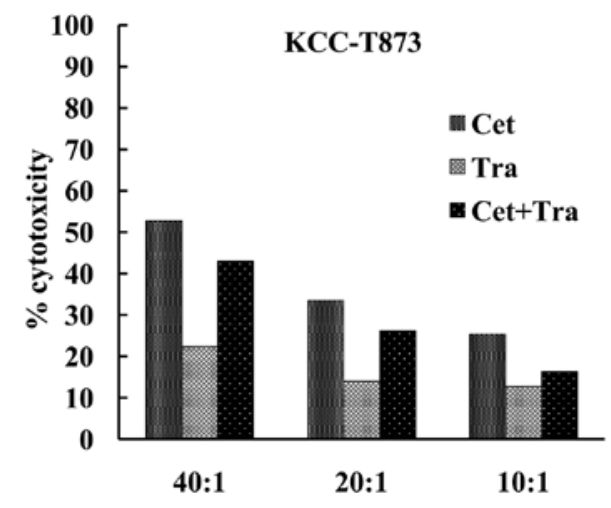

B

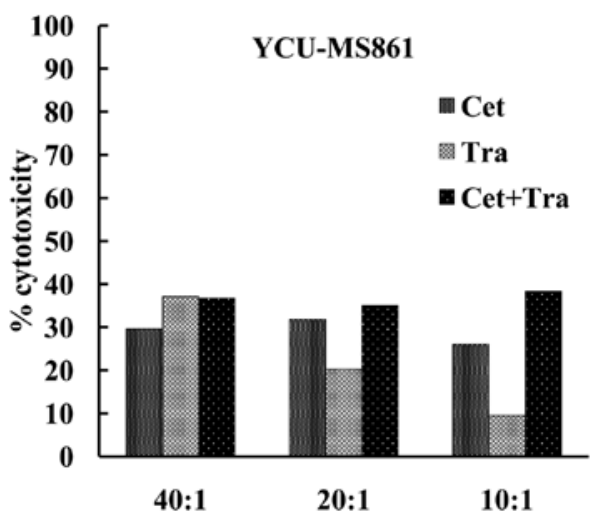

C

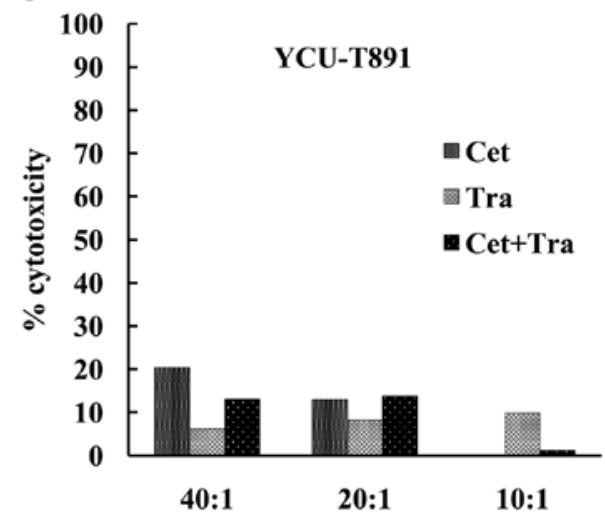

Figure 6. Cytotoxic assays in KCC-T873 (EGFR high, HER2 high), YCUMS861 (EGFR low, HER-2 moderate) and YCU-T891 (EGFR low, HER-2 low). PBMC was extracted from healthy donor A and cytotoxicity assays were performed at different $\mathrm{E} / \mathrm{T}$ ratios in the presence of cetuximab at a concentration of $100 \mathrm{nM}$ and/or trastuzumab at a concentration of $1 \mu \mathrm{M}$. (A) KCC-T873 (EGFR high, HER2 high). (B) YCU-MS861 (EGFR low, HER-2 moderate). (C) YCU-T891 (EGFR low, HER-2 low). Both cetuximab and trastuzumab alone showed ADCC in the 3 cell lines, but significant combination effect was not observed in any experimental model.

previously reported that HER-targeting therapy with gefitinib and trastuzumab combination is effective for HNSCC in vitro (4). Hence, we examined the antitumor effect of combined treatment with cetuximab and trastuzumab in vitro.

We have previously reported the effect of combined gefitinib and trastuzumab treatment on HNSCC (4). In the present
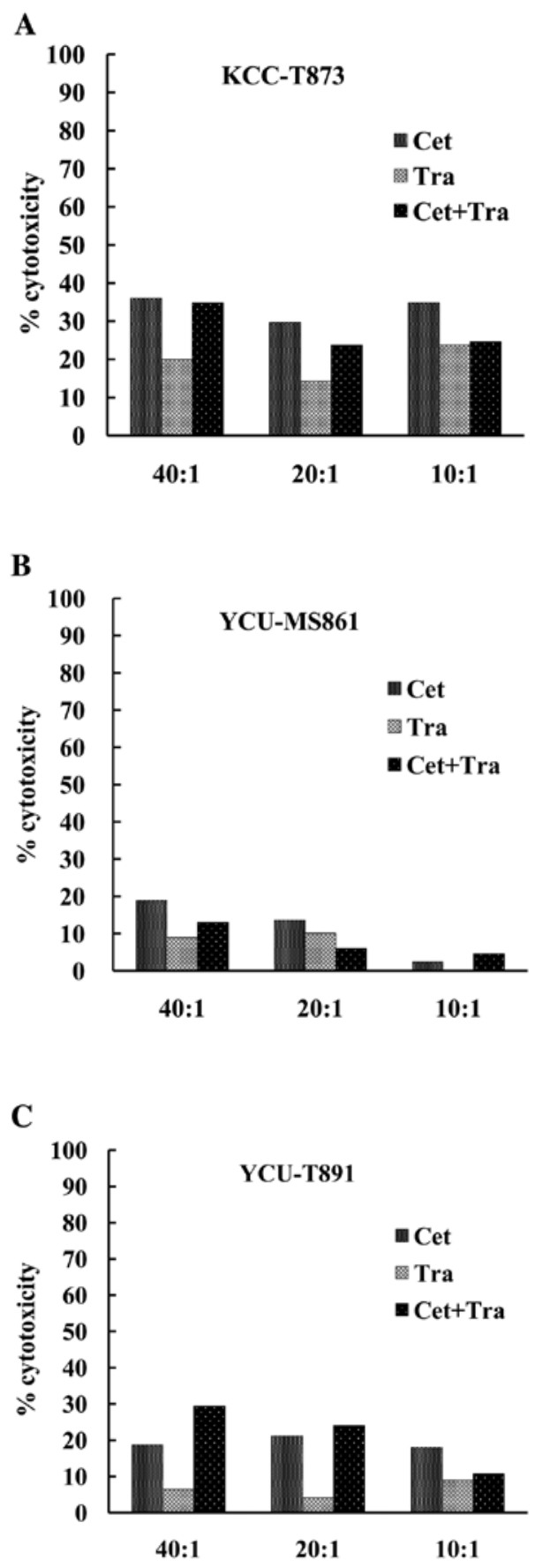

Figure 7. Cytotoxic assays in KCC-T873 (EGFR high, HER2 high), YCUMS861 (EGFR low, HER-2 moderate) and YCU-T891 (EGFR low, HER-2 low). PBMC was extracted from healthy donor $\mathrm{B}$ and cytotoxicity assays were performed at different $\mathrm{E} / \mathrm{T}$ ratios in the presence of cetuximab at a concentration of $100 \mathrm{nM}$ and/or trastuzumab at a concentration of $1 \mu \mathrm{M}$. (A) KCC-T873 (EGFR high, HER2 high). (B) YCU-MS861 (EGFR low, HER-2 moderate). (C) YCU-T891 (EGFR low, HER-2 low). Both cetuximab and trastuzumab alone showed ADCC in the 3 cell lines, but significant combination effect was not observed in any experimental model.

study, we evaluated the antiproliferative effect of trastuzumab alone on the same HNSCC cell lines; the results suggested that trastuzumab alone exerted very few antiproliferative effects on HNSCC (Fig. 4). Further, the antiproliferative effect of trastuzumab alone were weaker than those of cetuximab evaluated by the same method and using the same cell lines (24). In this 
study, we combined cetuximab and trastuzumab treatment and obtained significant growth inhibition in 8 out of 16 cell lines (Table I). Therefore, with regard to the antiproliferative effect of drugs on EGFR and HER-2, combined cetuximab and trastuzumab treatment will be effective on HNSCC. However, we found that only 2 cell lines exhibited $50 \%$ growth inhibition as compared to controls and the antiproliferative effect of combined treatment was not strong. In our previous study, we had evaluated antiproliferative effect of gefitinib by MTT method using the same 16 cell lines and obtained the $\mathrm{IC}_{50}$ values of gefitinib for all cell lines (4). Therefore, combined cetuximab and trastuzumab treatment showed significant antiproliferative effect such as EGFR blockage; however, growth inhibition rate by gefitinib alone was lower as compared to its antiproliferative effect evaluated by the same method using the same HNSCC cell lines (4).

To determine the correlation between the antiproliferative effect of combined cetuximab and trastuzumab treatment and HER expression, we analyzed the levels of HER-2, HER-3 and HER-4 mRNA in 16 HNSCC cell lines (Figs. 1-3). Previously we reported the expression levels of EGFR mRNA in HNSCC cell lines (24). All cell lines expressed mRNA for EGFR, HER-2 and HER-3; 12 cell lines expressed mRNA for HER-4; and 4 cell lines did not express mRNA for HER-4. Probably, the expression of both EGFR and HER-2 is required for the cetuximab and trastuzumab treatment to be effective; however, mRNA expression levels of EGFR and HER-2 were not correlated with the efficacy of combined cetuximab and trastuzumab therapy (Fig. 5A and B). HER-2 appears to function primarily as a heterodimerization partner for other HER family members (12); the expression levels of HER-3 and HER-4 also showed no correlation with the efficacy of combined cetuximab and trastuzumab therapy (Fig. 5C and D).

Cohen et al sequenced the EGFR TK domain and HER-2 in 8 gefitinib-responsive HNSCC patients (33). They did not find any mutation in EGFR but a single mutation was detected in HER-2. Accordingly, the authors suggested that some HNSCC patients responded to gefitinib due to mutations in $H E R-2$ rather than EGFR. In the present study, we did not observe mutation of $H E R-2$ in any cell line, suggesting that the mutation status of $H E R-2$ does not provide any additional information on the efficacy of combined cetuximab and trastuzumab treatment.

The role of IgG1 antibody in the killing of cancer cells by activating CDC and/or ADCC process is well established and experimentally proven. The in vivo antitumor effect of rituximab in B cell lymphoma, trastuzumab in breast and gastric cancer or cetuximab in colon cancer and HNSCC are mainly due to ADCC. These IgG1 monoclonal antibodies induce ADCC by blocking the human Fc receptor, i.e., IgG1 antibody binds the Fc receptors expressed by NK cells, monocytes and granulocytes $(17,34)$. Cetuximab-mediated ADCC has been reported in HNSCC (35-37); we have reported that cetuximab-induced ADCC and the antiproliferative effect by the MTT assay in HNSCC cells (24). However, trastuzumab-mediated ADCC in HNSCC has not been reported yet. Our data show that trastuzumab-induced ADCC by PBMC, obtained from two healthy donors, in all 3 cell lines. Kawaguchi et al reported that the combined cetuximab and trastuzumab therapy induced ADCC in esophageal cancer; however, 1 cell line showed marginal additional effects and 2 cell lines showed no significant effect (38). Similarly, in this study, the combined cetuximab and trastuzumab treatment did not exerted significant additional ADCC in the 3 cell lines as compared to the treatment with cetuximab or trastuzumab alone (Figs. 6 and 7).

On the basis of the reasons stated above, we concluded that the combination treatment with antibodies against both EGFR and HER-2 showed significant additional antiproliferative effect but no additional effect on ADCC. Previously we reported the effect of combined gefitinib and trastuzumab treatment on HNSCC cell lines in vitro (4).

Trastuzumab alone exhibited very slight antiproliferative effect. Combined treatment with antibodies for EGFR and HER-2 showed additional significant effect, but the total antiproliferative effect was not strong. With regard to ADCC, antibodies against EGFR or HER-2 alone exhibited IgG1mediated ADCC, but the combination treatment had no additional effect. These results suggest that combined treatment with molecular targeted drugs such as TKI for EGFR and IgG1 antibody for EGFR or HER2 may be effective owing to the antiproliferative effect and ADCC in HNSCC.

\section{Acknowledgements}

This study was supported by the Yokohama Foundation for Advancement of Medical Science (N.K.).

\section{References}

1. Reuter CW, Morgan MA and Eckardt A: Targeting EGF-receptorsignalling in squamous cell carcinomas of the head and neck. $\mathrm{Br}$ J Cancer 96: 408-416, 2007.

2. Forastiere AA, Goepfert H, Maor M, et al: Concurrent chemotherapy and radiotherapy for organ preservation in advanced laryngeal cancer. N Engl J Med 349: 2091-2098, 2003.

3. Cohen EE, Kane MA, List MA, et al: Phase II trial of gefitinib $250 \mathrm{mg}$ daily in patients with recurrent and/or metastatic squamous cell carcinoma of the head and neck. Clin Cancer Res 11: 8418-8424, 2005.

4. Kondo N, Ishiguro Y, Kimura M, et al: Antitumor effect of gefitinib on head and neck squamouscell carcinoma enhanced by trastuzumab. Oncol Rep 20: 373-378, 2008.

5. Harrington KJ, El-Hariry IA, Holford CS, et al: Phase I study of lapatinib in combination with chemoradiation in patients with locally advanced squamous cell carcinoma of the head and neck. J Clin Oncol 27: 1100-1107, 2009.

6. Kondo N, Tsukuda M, Ishiguro Y, et al: Antitumor effects of lapatinib (GW572016), a dual inhibitor of EGFR and HER-2, in combination with cisplatin or paclitaxel on head and neck squamous cell carcinoma. Oncol Rep 23: 957-963, 2010.

7. Siena S, Sartore-Bianchi A, Di Nicolantonio F, Balfour J and Bardelli A: Biomarkers predicting clinical outcome of epidermal growth factor receptor-targeted therapy in metastatic colorectal cancer. J Natl Cancer Inst 101: 1308-1324, 2009.

8. Bonner JA, Harari PM, Giralt J, et al: Radiotherapy plus cetuximab for locoregionally advanced head and neck cancer: 5 -year survival data from a phase 3 randomised trial, and relation between cetuximab-induced rash and survival. Lancet Oncol 11: 21-28, 2010.

9. Eisbruch A, Blick M, Lee JS, Sacks PG and Gutterman J: Analysis of the epidermal growth factor receptor gene in fresh human head and neck tumors. Cancer Res 47: 3603-3605, 1987.

10. Santini J, Formento JL, Francoual M, Milano G, Schneider M, Dassonville O and Demard F: Characterization, quantification, and potential clinical value of the epidermal growth factor receptor in head and neck squamous cell carcinomas. Head Neck 13: 132-139, 1991. 
11. Klapper LN, Vaisman N, Hurwitz E, Pinkas-Kramarski R, Yarden $\mathrm{Y}$ and Sela M: A subclass of tumor-inhibitory monoclonal antibodies to ErbB-2/HER2 blocks crosstalk with growth factor receptors. Oncogene 14: 2099-2109, 1997.

12. Graus-Porta D, Beerli RR, Daly JM and Hynes NE: ErbB-2, the preferred heterodimerization partner of all ErbB receptors, is a mediator of lateral signaling. EMBO J 16: 1647-1655, 1997.

13. Khademi B, Shirazi FM, Vasei M, et al: The expression of $\mathrm{p} 53$, c-erbB-1 and c-erbB-2 molecules and their correlation with prognostic markers in patients with head and neck tumors. Cancer Lett 184: 223-230, 2002.

14. Alaoui-Jamali MA, Paterson J, Al Moustafa AE and Yen L: The role of ErbB-2 tyrosine kinase receptor in cellular intrinsic chemoresistance: mechanisms and implications. Biochem Cell Biol 75: 315-325, 1997.

15. Rogers SJ, Harrington KJ, Rhys-Evans P, O-Charoenrat P and Eccles SA: Biological significance of c-erbB family oncogenes in head and neck cancer. Cancer Metastasis Rev 24: 47-69, 2005.

16. Hara M, Nakanishi H, Tsujimura K, Matsui M, Yatabe Y, Manabe $T$ and Tatematsu M: Interleukin-2 potentiation of cetuximab antitumor activity for epidermal growth factor receptor-overexpressing gastric cancer xenografts through antibody-dependent cellular cytotoxicity. Cancer Sci 99: 1471-1478, 2008.

17. Kimura H, Sakai K, Arao T, Shimoyama T, Tamura T and Nishio K: Antibody-dependent cellular cytotoxicity of cetuximab against tumor cells with wild-type or mutant epidermal growth factor receptor. Cancer Sci 98: 1275-1280, 2007.

18. Baselga J, Tripathy D, Mendelsohn J, et al: Phase II study of weekly intravenous recombinant humanized anti-p185HER2 monoclonal antibody in patients with HER2/neu-overexpressing metastatic breast cancer. J Clin Oncol 14: 737-744, 1996.

19. Sliwkowski MX, Lofgren JA, Lewis GD, et al: Non-clinical studies addressing the mechanism of action of trastuzumab (Herceptin). Semin Oncol 4 (Suppl 12): S60-S70, 1999.

20. Kono K, Takahashi A, Ichihara F, Sugai H, Fujii H and Matsumoto Y: Impaired antibody-dependent cellular cytotoxicity mediated by herceptin in patients with gastric cancer. Cancer Res 62: 5813-5817, 2002.

21. Sheikh Ali MA, Gunduz M, Nagatsuka H, et al: Expression and mutation analysis of epidermal growth factor receptor in head and neck squamous cell carcinoma. Cancer Sci 99: 1589-1594, 2008.

22. Sartori G, Cavazza A, Bertolini F, et al: A subset of lung adenocarcinomas and atypical adenomatous hyperplasia-associated foci are genotypically related: an EGFR, HER2, and K-ras mutational analysis. Am J Clin Pathol 129: 202-210, 2008.

23. Xu JM, Han Y, Duan HQ, et al: EGFR mutations and HER2/3 protein expression and clinical outcome in Chinese advanced non-small cell lung cancer patients treated with gefitinib. J Cancer Res Clin Oncol 135: 771-782, 2009

24. Kondo N, Tsukuda M, Taguchi T, et al: Gene status of head and neck squamous cell carcinoma cell lines and cetuximabmediated biological activities. Cancer Sci 102: 1717-1723, 2011.

25. Choong NW and Cohen EE: Epidermal growth factor receptor directed therapy in head and neck cancer. Crit Rev Oncol Hematol 57: 25-43, 2006.
26. Cohen EE, Lingen MW and Vokes EE: The expanding role of systemic therapy in head and neck cancer. J Clin Oncol 22: $1743-1752,2004$

27. Pegram MD, Lipton A, Hayes DF, et al: Phase II study of receptor-enhanced chemosensitivity using recombinant humanized anti-p185HER2/neu monoclonal antibody plus cisplatin in patients with HER2/neu-overexpressing metastatic breast cancer refractory to chemotherapy treatment. J Clin Oncol 16: 2659-2671, 1998

28. Pinkas-Kramarski R, Soussan L, Waterman H, et al: Diversification of Neu differentiation factor and epidermal growth factor signaling by combinatorial receptor interactions. EMBO J 15: 2452-2467, 1996.

29. Warburton C, Dragowska WH, Gelmon K, et al: Treatment of HER-2/neu overexpressing breast cancer xenograft models with trastuzumab (Herceptin) and Gefitinib (ZD1839): drug combination effects on tumor growth, HER-2/neu and epidermal growth factor receptor expression, and viable hypoxic cell fraction. Clin Cancer Res 10: 2512-2524, 2004.

30. Nakamura H, Takamori S, Fujii T, Ono M, Yamana H, Kuwano M and Shirouzu K: Cooperative cell-growth inhibition by combination treatment with ZD1839 (Iressa) and trastuzumab (Herceptin) in non-small cell lung cancer. Cancer Lett 230: 33-46, 2005.

31. Formento P, Hannoun-Levi JM, Fischel JL, Magne N, EtienneGrimaldi MC and Milano G: Dual HER 1-2 targeting of hormone-refractory prostate cancer by ZD1839 and trastuzumab. Eur J Cancer 40: 2837-2844, 2004.

32. Fukutome M, Maebayashi K, Nasu S, Seki K and Mitsuhashi N: Enhancement of radiosensitivity by dual inhibition of the HER family with ZD1839 ('Iressa') and trastuzumab ('Herceptin'). Int J Radiat Oncol Biol Phys 66: 528-536, 2006.

33. Cohen EE, Lingen MW, Martin LE, et al: Response of some head and neck cancers to epidermal growth factor receptor tyrosine kinase inhibitors may be linked to mutation of ERBB2 rather than EGFR. Clin Cancer Res 11: 8105-8108, 2005.

34. Roda JM, Joshi T, Butchar JP, McAlees JW, Lehman A, Tridandapani S and Carson WE: The activation of natural killer cell effector functions by cetuximab-coated, epidermal growth factor receptor positive tumor cells is enhanced by cytokines. Clin Cancer Res 13: 6419-6428, 2007.

35. Taylor RJ, Chan SL, Wood A, Grandis JR, Gooding WE, Ferrone S and Ferris RL: Fc $\gamma$ RIIIa polymorphisms and cetuximab induced cytotoxicity in squamous cell carcinoma of the head and neck. Cancer Immunol Immunother 58: 997-1006, 2009.

36. Lopez-Albaitero A, Lee SC, Morgan S, et al: EGFR expression level in cetuximab mediated, NK cell dependent in vitro cytotoxicity of head and neck squamous cell carcinoma cells. Cancer Immunol Immunother 58: 1853-1864, 2009.

37. Lopez-Albaitero A and Ferris RL: Immune activation by epidermal growth factor receptor specific monoclonal antibody therapy for head and neck cancer. Arch Otolaryngol Head Neck Surg 133: 1277-1281, 2007.

38. Kawaguchi Y, Kono K, Mimura K, Sugai H, Akaike H and Fujii H: Cetuximab induce antibody-dependent cellular cytotoxicity against EGFR-expressing esophageal squamous cell carcinoma. Int J Cancer 120: 781-787 2007. 